\title{
Chaotic Dynamics of a Non-linear Electronic Circuit
}

\author{
T.Sivasakthi, S.Gayathri, K.Jeyapiriya
}

\begin{abstract}
A very simple nonlinear parallel/series, non autonomous/ autonomous circuits that has Chua's diode which is its only nonlinear element, shows a wide variety of different features such as chaos, quasi periodicity, period adding/doubling, window etc is proposed. the effect of an external periodic varioations on the Chua's piecewise linear circuit is studied. Under the action of such a force this circuit exhibits a large variety bifurcation sequences, including doubling of period, that adds to chaos regime.
\end{abstract}

KeyWords -Chaos in electronic circuit, Circuit model, Experimental Results of the Circuit, System Specifications.

\section{INTRODUCTION}

Dynamical system has two components: 1 . The initial value of the system defining the state of the origin of the system and 2. The dynamicity which explains the evolution of the system. Any dynamic system is described by the mathematical modeling using differential equation. The scientific philosophy which states that the basic of any mathematical philosophy is the differential equation. This originated with the discovery of calculus and it still continues to play the major role even today.

Dynamical systems theory produces a qualitative study of the differential equations. Another study is non linear dynamics. This helps us understanding any motions around us ie., vibration of a string, movement of planets around an orbit, the ripples or waves on the water surface, the changing patterns of weather conditions in an area.

Over 200 years from the beginning of this scientific philosophy there had been a tremendous success in designing the rules of the earth but is limited to only very few rules in arriving at solutions. Some motions that are regular in pattern like the oscillations of a pendulum can be easily understood and explained whereas some irregular patterns such as waterfall cannot be easily analyzed and fail to fit to any such rules.

He says that the things that appear very meager or unnoticed to people produces a noticeable effect. To understand the universe and the scenario at present, one needs to know the initial situation and nature's laws and its behavior. Though the laws of nature gives a clear picture and understanding but it still provides only approximate initial conditions. With this few and approximate initial conditions; one may predict the future situation with the knowledge of the laws of nature.

Revised Manuscript Received on December 5, 2019.

Mr.T.Sivasakthi, Assistant Professor, Department of ECE, Sri Sai Ram Engineering College, Chennai, Tamil Nadu,India.

Ms.K.Jeyapiriya, Assistant Professor, Department of ECE, Sri Sai Ram Engineering College, Chennai, Tamil Nadu,India.

Ms. S.Gayathri , Assistant Professor, Department of ECE, Sri Sai Ram Engineering College, Chennai, Tamil Nadu, India.
This prediction may produce a small error which accumulates to produce a huge error later. This makes prediction an impossible method. Poincare then discovered a sensitive dependence on initial conditions. This was welcomed by many big scientists and

mathematicians. Many others have found this chaotic properties in many non linear systems.

Here, one must understand the difference between the non linear dynamics and the dynamical system theory. Non linear dynamics is interdisciplinary of science and mathematics whereas dynamical system theory is mathematical. Thus the analysis of non linear systems gains its importance by various scientists working in different areas. A meteorologist discovered in an attempt to know the unpredictability of weather. A biologist came out with the study of the quadratic map in an attempt to understand the population dynamics. Every scientists work on their non linear systems making this discipline alive. To know a nonlinear dynamical system one must first look at an example of linear dynamical system. A linear dynamical system is one in which the dynamic rule and system variables are linearly proportion to one another. Linear systems can be sub divided to produce smaller modules and their individual responses can be summed up to obtain a complete solution.

\section{CHAOS IN ELECTRONIC CIRCUIT}

A simple circuit helps one to i) to construct the system easily, ii) the parameters can be observed using a simple software simulation technique, iii) it proves the circuit is chaotic.

The validity of the followings statements are needed to be questioned:

Oscillation $=$ periodic

Noise $=$ nondeterministic .

Now anyone can confidently prove that both the statements are not true. This chapter provides an evidence and a rigorous proof for the above mentioned two statements. Even a very simple system can prove to be evidence of the above mentioned statements. The practical implementation of the system shows the systems are of lower order and deterministic systems. The unpredictability of the system lies in the way that when there is a very small change in the initial condition, it leads to a different conclusion. Periodic oscillators are said to be predictable when it concludes to same periodic orbit even under different initial conditions. There may also occur deterministic noise under practical environment when executed experimentally. In this paper, the word "chaotic" means "the system that has no periodic oscillations. 


\section{UTILIZATION OF CHAOTIC SIGNALS}

Utilization of chaotic signals in,

1. Communication system,

2. Biomedical engineering,

3. Radars,

4. Decoding algorithms,

5. Synchronization phenomena between two coupled oscillators,

6. Musical Signals from Chua's Circuit. are some of the applications of chaotic signals.

\section{A. An Idea to Generate Chaos in Electronic Circuit}

This section concentrates on the Linear system theory. This theory explains the characteristics of the sustained oscillations of the ature. The system is designed to produce two works from linear systems with simple harmonics to non linear systems with chaos. The basic goal is to stimulate one to understand the basic concepts of the oscillation principles and to extend further the knowledge to non linear dynamic system that produces chaos. To demonstrate the chaos, a circuit that is autonomous or a non autonomous consisting of passive circuit elements is needed and it must contain a minimum of one non linear element, one locally active resistor and a minimum of 3energy conserving elements. All these conditions mentioned here are satisfied by the Chua's circuit. Moreover, it has been proven mathematically the chaos in a system. This circuit is also economical and produces desired bifurcation differences and chaos. Linear circuit theory exposes a person from a periodic output produced by a linear passive circuit to a complex Chua's circuit containing chaos. The following are the various parameters obtained from the worked examples and background theory,

i) Equilibrium points,

ii) Steady-state solutions

iii) Limit cycles

iv) Bifurcations

v) Stability

vi) Chaos.

With the foundation of this theoretical concept, a parallel and linear RLC resonant circuit is constructed and is developed from a two $2^{\text {nd }}$ order piecewise and linear circuits to a Chua's circuit.

\section{B. Circuit Model}

The realization of the proposed simple non-autonomous circuit is depicted in fig. it consists of two nonlinear inductors, a linear capacitor, linear negative conductance, two nonlinear diodes and a sinusoidal voltage source. The basic difference between the Chua's circuit and the present circuit is that here Chua's diode is replaced by a of a $p-n$ junction diode and a negative conductance. The V-I characteristics of the diode is approximated by the two segment

piece wise linear function.

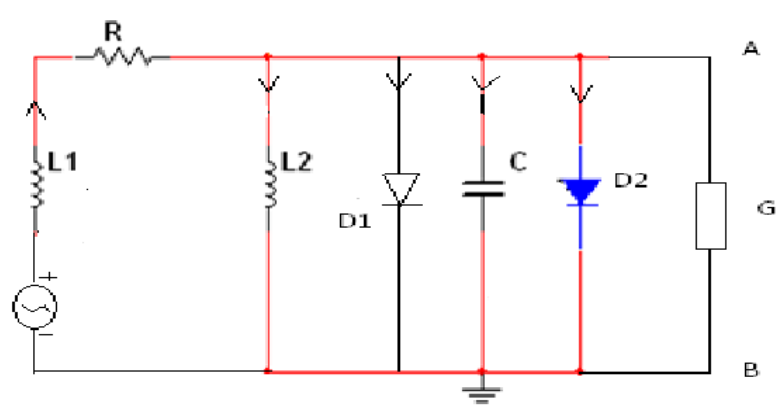

Fig 1: Realization of the proposed non autonomous circuit

\section{Experimental Setup of the Circuit}

The entire block diagram of the experimental set up is shown fig. For the experimental study, we are getting the external oscillatory signal from the function generator $(5.5$ $\mathrm{KHz}$ ). The dynamical behavior of the circuit is observed using the help of cathode ray oscilloscope. To start our experiment, we fix the values of each element. This we do from our experience about the values available in the literature. Thus the inductance and capacitance are chosen in the range of $\mathrm{mH}$ and $\mathrm{nF}$ respectively. The resistance and frequency are chosen in the range of $\operatorname{ohm}(\Omega)$ to kilo $\operatorname{ohm}(\mathrm{k} \Omega)$ and hertz $(\mathrm{Hz})$ to kilo hertz (KHZ) respectively. We carry out the experiments by fixing the value of elements except the amplitude of the external source signal, which is actually varied during the experiment. The values are given by $\mathrm{L} 1=101.3 \mathrm{mH}, \mathrm{L} 2=61.9 \mathrm{mH}, \mathrm{C}=10 \mathrm{nF}, \mathrm{R}=492$ $\mathrm{ohm}, \mathrm{G}=0.45 \mathrm{~ms}$ and the frequency $\mathrm{f}=5.5 \mathrm{KHz}$. Here as mentioned the amplitude of the forced signal is the variable one. Thus the amplitude is treated as the control parameter for the circuit. Fig shows the laboratory realization of negative conductance $G$. In this circuit, an operational amplifier (op-amp) IC741 is used with three $2 \mathrm{~K}$ linear resistors. The positive $(V+=+9 V)$ and negative $(V-=-9 V)$ voltage are given to pin numbers

seven and four of the op-amp IC741 respectively. A and B are the positive and negative terminals of the negative conductance $\mathrm{G}$. The X-Y terminal of CRO is connected to trace the attractors projected in the CRO screen. We select the circuit parameters negative conductance as $\mathrm{R} 1=\mathrm{R} 2=\mathrm{R} 3=2 \mathrm{~K} \Omega$. The whole experimental set up is done at in computer simulation software PSPICE.

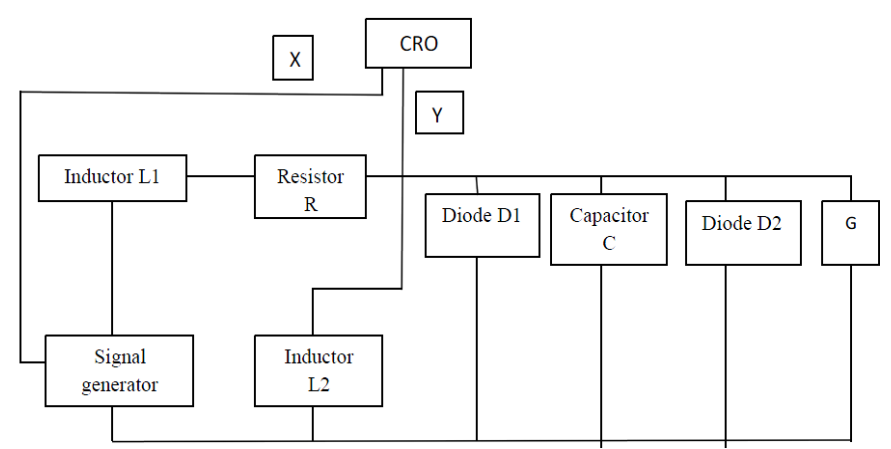

Fig 2: Block diagram of the total experimental setup of the circuit 


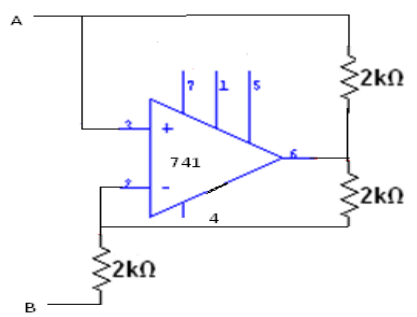

Fig 3: Laboratory Realization of negative conductance

\section{Experimental Results of the Circuit}

By increasing the amplitude of the periodic signal from $50 \mathrm{mV}$, the circuit is found to exhibit a sequence of bifurcations starting from quasi periodic attractor, chaos, period 1, period window, and boundary crisis etc. These outputs are tracked by CRO screen for different range of the amplitude of the external periodic signal. The experimental observations are found in fig.4. For small values $\mathrm{F}(\sim 50 \mathrm{mV})$ quasi periodicity occurs. This quasi periodicity is stable for $50<\mathrm{F}<266 \mathrm{mV}$. As the amplitude increases to $\mathrm{F}=267 \mathrm{mV}$, there produces a unstable and quasi stability and period 1 orbit is born. This orbit is stable in the range amplitude $267<\mathrm{F}<330 \mathrm{mv}$. As the parameter $\mathrm{F}$ is further increased, period window is found to occur leading to onset chaos. Increase the amplitude of the signal F. It is noticed that the window orbit is found to be changed to period 2 orbit at $\mathrm{F}=360 \mathrm{mV}$ it is found to be stable in the range $360<\mathrm{F}<390$ $\mathrm{mV}$. When the amplitude is further increased, quasi periodicity is again take place at the amplitude $\mathrm{F}=420 \mathrm{mV}$ and is stable in the range of $420<\mathrm{F}<530 \mathrm{mV}$. The amplitude of the external periodic signal $\mathrm{F}$ is further increased the quasi periodicity orbit is changed to the period lorbit at $\mathrm{F}=540 \mathrm{mV}$ and it is stable in the range $40<\mathrm{F}<600$

$\mathrm{mV}$. Finally the system enters into a boundary crisis when the amplitude value is increased beyond $600 \mathrm{mV}$.

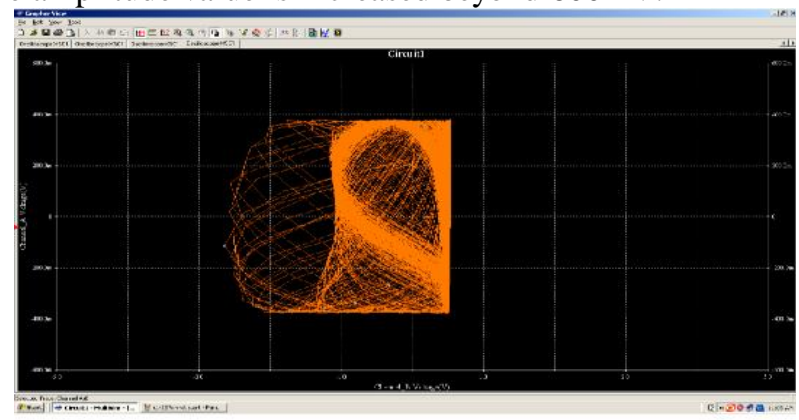

Fig 4: Quasiperiodicity $(50<\mathrm{F}<266 \mathrm{mV})$

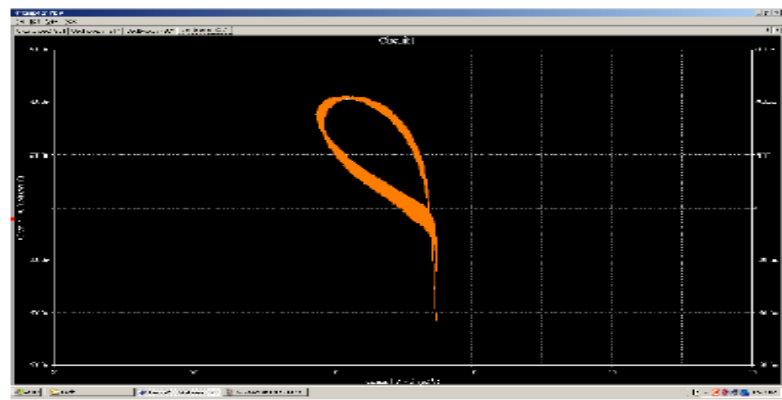

Fig 4.1: Period 1 or $1 T(267<\mathrm{F}<330 \mathrm{mV})$

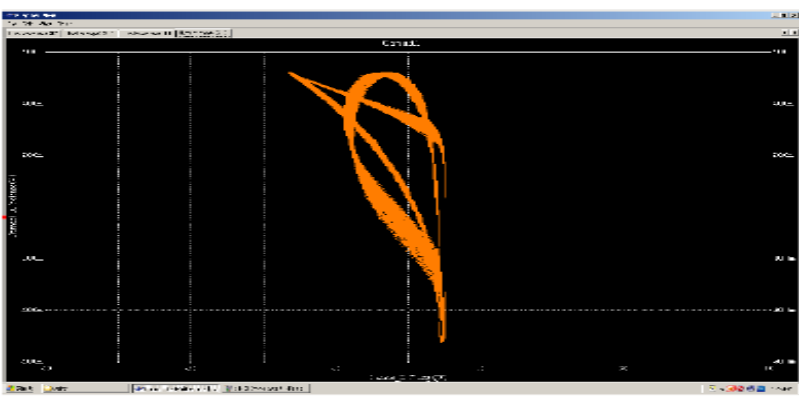

Fig 4.2: Period 2 or $2 \mathrm{~T}(360<\mathrm{F}<390 \mathrm{mV})$

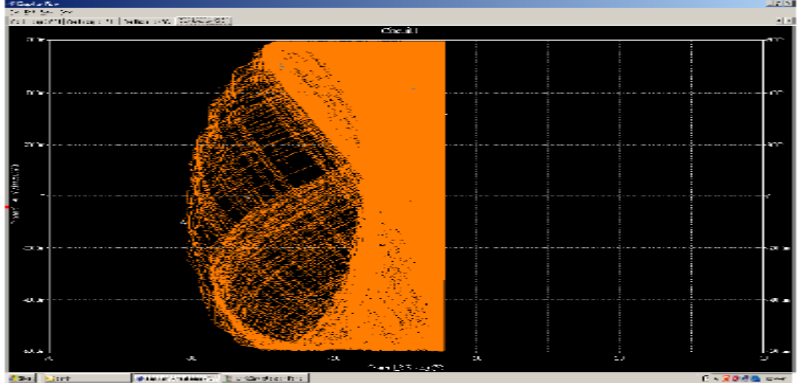

Fig 4.3: Quasiperiodicity $(420<F<530 \mathrm{mV})$

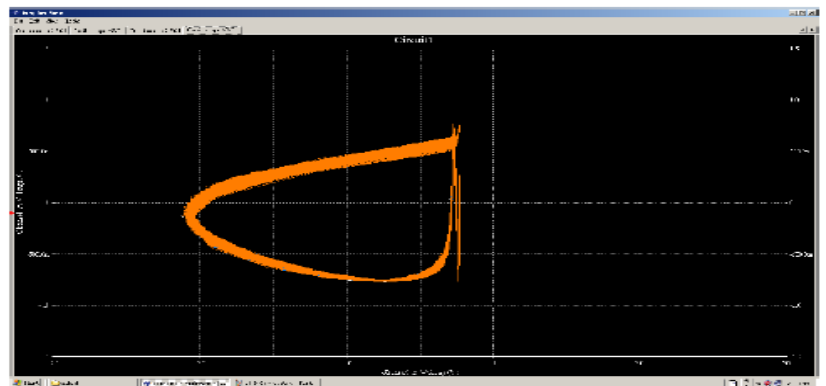

Fig 4.4: Period 1 or $1 T(540<F<600 \mathrm{mV})$

\section{E. Flow Diagram}
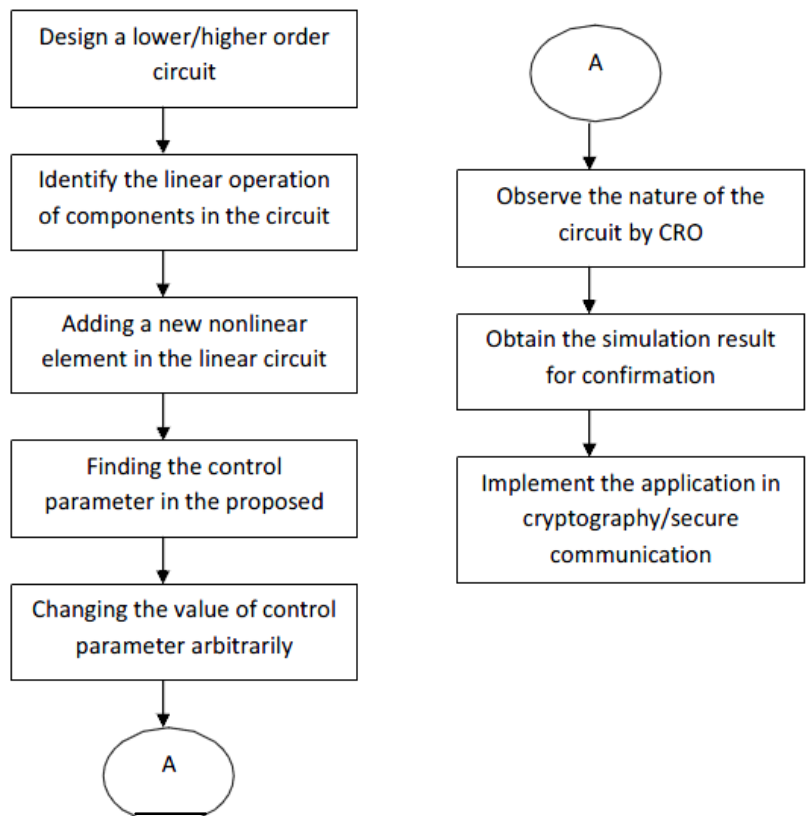

Fig 4: Problem Formulation and solution methodology

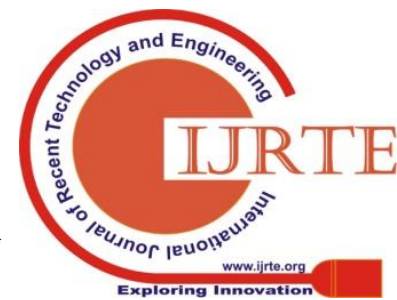




\section{CONCLUSION}

A simple non-autonomous, higher order chaotic circuit has been introduced. Under the influence of the external signal, and a new nonlinear element named p-n junction diode, we found rich variety nonlinear characteristics. Though many complex ubiquitous ways be available to chaos like periodic attractor, a simple electronic circuit with boundary crisis, one can work on various chaotic environments and their applications as well, that includes cascaded heterogeneous chaotic systems.

\section{REFERENCES}

1. T.Matsumato, L.O.Chua \& K.Tokumasu, (Aug 1986) "Double scroll via a Two-Transistor circuit" IEEE Tr.ans.circuit syst., vol CAS-33, pp.828-835,

2. K.Murali and M.Lakshmanan, (Apr 1992)"Effect of sinusoidal excitation on the chua's circuit" IEEE Tr.ans.circuit syst.,vol -39,NO-4, pp.2642-270,.

3. K.Murali and M.Lakshmanan,"Experimental chaos from nonautonomous electronic Circuits" Phil.Trans.R.Soc..Lond A(1995)353,33-46.

4. K.Thamilmaran, M.Lakshmanan and K.Murali, "Rich variety of bifurcation and chaos in Variant of murali-lakshmanan-chua circuit" International journal of bifurcation and Chaos, vol.10, No.7(2000)17811785 .

5. M.E Yalcin, J.A.K, suykens and J.vandwelle, (Mar 2000) ,"Experimental confirmation of 3- and 5- scroll attractors from generalized Chua's circuit” IEE Tr.ans.circuit syst-PART 1: FUNDAMENTAL THEORY AND APPLICATIONS.vol -47, NO-3, pp.425-429,

6. E.campos-canton and I.campos canton and J.s.Ganzale sales and F.cruz ordaz,"A parametrized family of single-double-triple-scroll chaotic oscillations"revista mexicana de fisca 54(6)411-415.

7. A.Balamurugan and V.sengodan, "Rich variety of bifurcation and chaos in a simple nonsource free electronic circuit with a diode" ,International journal of pure and applied physics ISSN 0973-1776 vol 6,No 1(2010),pp 63-69.

8. Bhratwaj muthuswamy \& Leon o,"Simplest chaotic circuit", International journal of bifurcation and chaos, vol.20, No.5 (2010)15071580 .

\section{AUTHORS PROFILE}

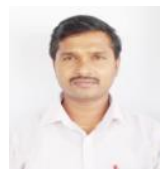

Mr.T.Sivasakthi, working as Assistant Professor in the Department of ECE, Sri SaiRam Engineering College and has a teaching experience of 8 years. He had Completed UG in Electronics and Communication Engineering and PG in Applied Electronics. His area of Interests includes Signal Communication. Processing, Fiber Bragg Grating Sensors, Optical Fiber

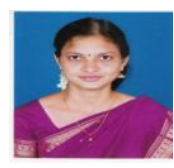

Ms.K.Jeyapiriya, working as Assistant Professor in the Department of ECE, Sri SaiRam Engineering College and has a teaching experience of 16 years. She has published several papers in International and National Journals and Conferences. Her area of Interests includes Image, Signal Processing and networking.

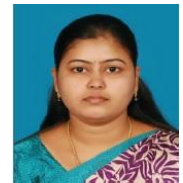

Ms. S.Gayathri , working as Assistant Professor in the Department of ECE, Sri SaiRam Engineering College and has a teaching experience of 10 years. She had Completed UG in Electronics and Communication Engineering and PG in Applied Electronics. Her area of Interests include Image and Signal Processing. 